\title{
Emotional and Functional Challenge in Core and Avant-garde Games
}

\author{
Tom Cole \\ Department of Computing, \\ Goldsmiths, \\ University of London \\ London, UK \\ t.cole@gold.ac.uk
}

\author{
Paul Cairns \\ Department of \\ Computer Science, \\ University of York \\ York, UK \\ paul.cairns@york.ac.uk
}

\author{
Marco Gillies \\ Department of Computing, \\ Goldsmiths, \\ University of London \\ London, UK \\ m.gillies@gold.ac.uk
}

\begin{abstract}
Digital games are a wide, diverse and fast developing art form, and it is important to analyse games that are pushing the medium forward to see what design lessons can be learned. However, there are no established criteria to determine which games show these more progressive qualities.

Grounded theory methodology was used to analyse language used in games reviews by critics of both 'core gamer' titles and those titles with more avant-garde properties. This showed there were two kinds of challenge being discussed - emotional and functional which appear to be, at least partially, mutually exclusive. Reviews of 'core' and 'avantgarde' games had different measures of purchase value, primary emotions, and modalities of language used to discuss the role of audiovisual qualities. Emotional challenge, ambiguity and solitude are suggested as useful devices for eliciting emotion from the player and for use in developing more 'avant-garde' games, as well as providing a basis for further lines of inquiry.
\end{abstract}

\section{Author Keywords}

Emotional; functional; challenge; ambiguity; solitude; grounded theory; digital games; art; avant-garde

\section{ACM Classification Keywords}

H.5.m. Information Interfaces and Presentation (e.g. HCI): Miscellaneous

\section{INTRODUCTION \\ "Our expectations of what a game should be have changed a lot over the years, and at this point, a game where you aren't near-constantly killing or at least phys- ically fighting things has become weird or unconven- tional." [9]}

Largely thanks to the advent of digital distribution and new mobile platforms, the range of digital games available has

Permission to make digital or hard copies of all or part of this work for personal or classroom use is granted without fee provided that copies are not made or distributed for profit or commercial advantage and that copies bear this notice and the full citation on the first page. Copyrights for components of this work owned by others than ACM must be honored. Abstracting with credit is permitted. To copy otherwise, or republish, to post on servers or to redistribute to lists, requires prior specific permission and/or a fee. Request permissions from permissions@ acm.org.

CHI PLAY 2015, October 03-07, 2015, London, United Kingdom

(c) 2015 ACM. ISBN 978-1-4503-3466-2/15/10\$15.00

DOI: http://dx.doi.org/10.1145/2793107.2793147 never been greater or so diverse and the appeal of games has broadened to the extent that some popular critics have declared the stereotypical 'core gamer' identity (the market segment defined by video game publishers as being historically the most committed to the hobby and therefore the most profitable) as outmoded and defunct [4]. Games are at a point where many (from both academia [21] and journalism [40]) are calling for them to be taken seriously and come under greater aesthetic scrutiny. However, despite the significant skill and craft involved in creating games, a number of commentators have also concluded that not all games show artistic merit [38] [3] on the basis that many are developed and marketed primarily as entertainment products (rather than art) to the traditional 'core gamer' market. Which titles then, in the words of the quote above, are non-core and therefore "weird and unconventional'? Definitions of avant-garde works in other media, such as film [29] [7] are useful in part, but are unable to account for all the possibilities afforded by the interactive qualities of video-games. There are recent attempts to define avant-garde videogames [37] [36], but little work is available on what criteria should be used to identify a title that is more avant-garde and how it would feel to play one. Are there any patterns to be discerned in how these more avantgarde titles differ to those for more the traditional core gamer demographic?

The popular games press sometimes tends to cast this as a difference between the 'triple-A' sector (where budgets and production values are very high) and the 'indie' sector (where budgets are lower and financial risk much less, often resulting in more creative and novel designs). However, many indie games still follow established conventions in the medium and are no more avant-garde than triple-A titles. It is suggested here it would be more productive to focus on the kind of experience that a game attempts to offer and examine first-hand accounts of the gameplay experience - in this case, video game reviews. If common motifs across core and/or avant-garde titles can be found they can be highlighted for further investigation for both game development and academic study. It should be noted that the designation of a game as 'core' in this investigation is not a quality judgement and does not infer that it lacks artistic merit or skill just that it is less experimental in ideas, methods or subject matter. 


\section{METHOD}

The approach taken was to analyse what video-games critics had written about a selection of titles. Previous analysis of video-games reviews has focused on elucidating the core user experience of playing video-games [11], but doesn't offer any clues as to how core and avant-garde games may be identified. Using Grounded Theory as initially outlined by Strauss and Corbin [39] and further refined by Adams, Lunt and Cairns [2] the aim was to investigate what differentiated core games from avant-garde games by looking at how reviewers reported their gaming experiences. Analysis focused on the style of language used, what aspects of the gaming experience were mentioned and how much was written about those aspects.

Reviews of a range of 14 games (from 3 categories) were selected for analysis. Due to lack of academic discourse this selection is based upon general understanding in the popular press and media discussion around these games. To The Moon (TTM), Gone Home, Dear Esther, The Vanishing of Ethan Carter (VoEC) and Papers Please are all critically acclaimed for pushing the boundaries of the medium and having more avant-garde aspirations. Gears of War 3 (GOW3), Call of Duty: Modern Warfare 2 (COD:MW2), Grand Theft Auto $V(G T A V)$, Far Cry 4 and Destiny are all highly successful blockbuster or 'triple-A' titles. Bioshock, Journey, Spec Ops: The Line (SO:TL) and The Last of Us (TLoU) are all innovative triple-A titles which, each in their own way, experimenting with what big-budget games can achieve. One of the aims of grounded theory is to choose a sample that stretches and tests the theoretical boundaries of interpretation of the data. For this reason the selection of games was intentionally diverse and broad.

The reviews were gathered from www.metacritic.com (a website that aggregates user and critic media reviews) filtering for critics reviews and ranked in order of 'most active' (the number of reviews submitted by the contributing website/magazine). Critics' reviews (rather than User reviews) were selected on the basis that, since they are writing in a professional capacity, they are more likely to have played the game for longer before passing judgement, show greater care and accountability for the words they write and therefore be more likely to deliver a higher quality critique. Whilst problems with preference bias could potentially be found e.g. some critics writing on certain types of games only, nonprofessional review writers would show the same bias and so this is not important in the context of the current investigation. Reviews were accessed and catalogued as long as they were written in English and available on-line. Using this process at least 10 reviews for each game were read and analysed - in excess of 150 reviews in total. Analysis was conducted using open coding for style of language used, expressions of what aspects were important about the game, topics covered and how long was spent talking about the different features of the game-playing experience. Subsequent axial coding and reanalysis was performed which allowed clusters of meaning to be grouped via selective coding into core and sub-categories for discussion.

\section{RESULTS}

Contrary to what many commentators (or major industry figures[23])may think, the premise, background story and description of the player character(s) for each game took up significant parts of the word count of every review. Story is clearly important to players, if only to establish context for the actions taken in game - even if it not highlighted as a primary feature of the experience. Reviewers also wrote at length about the quality, depth and complexity of the world the game took place in. Across all titles story and worldbuilding were key features of the gaming experience - even though the quality and centrality of the narrative to the gameplay experience varied. Despite it's universal importance here, the complexities of storytelling in interactive games is both well discussed elsewhere (e.g. [26], [30], [25]) and is outside the scope of this review.

Beyond this it became clear that two distinct sets of standards and style of language were being used to describe the gaming experience of the reviewer. The clearest trend seen was the difference between aspects linked to the 'functional challenge' offered by some titles and those linked to the 'emotional challenge' offered by others. These aspects included differing notions of what constitutes value in a game purchase, the primary emotion(s) experienced and the way the audiovisual components were described. Due to space constraints, quotes are representative of data and are not intended to be comprehensive.

\section{Challenge: Functional vs. Emotional}

Challenge, in some form, is central to the gaming experience. Yet until recently, the majority of challenges presented by games to their players are of the functional type - where dexterity and skill with the controls or strategy is used to overcome challenges thrown at the player (e.g. environmental traversal, combat, logical puzzles etc.) and to resolve emotions of frustration to fiero (an Italian word that literally translates as 'proud', here used to mean personal triumph as per Lazarro's usage [24]). It is this concept of functional challenge that is still presented to, and desired by, players of traditional core games such as Destiny and COD:MW2. Core mechanics are covered with enthusiasm and in great detail. The quality of enemy and friendly AI are also frequently discussed.

When faced with games with a majority functional challenge component, reviewers are more concerned with technical and mechanical considerations such as how the character moves, what powers they have, what actions they perform, what weapons they can wield and, often, how powerful the game makes the player feel. The reviewers' focus is, "What can I do?", and the core pleasures obtained are that of power and 'hard fun' [24].

“... the ability to sprint and slide in addition to each class' mobility skills, which include gliding, double jumping, and even short-range teleportation. Combined with how powerful grenades and melee attacks feel, 
thanks again to class skills that modify them, this extra mobility allows you to engage foes in a wider variety of ways." [19]

This contrasts strongly with accounts of playing avant-garde games, where mechanics were mentioned but seldom dwelt upon for long. Here the focus is more on the narrative, story and themes of the piece. Here, the reviewer's primary focus is, "How do I feel?" This is achieved by leaving parts of the experience ambiguous, confronting them with difficult material or by use of strong characters, story and good writing. This presents the player with an 'emotional challenge' that is overcome not with skill and dexterity, but with a cognitive effort not dissimilar to Schopenhauers notion of the aesthetic experience of the sublime. [35] The core pleasure here for the player is the resolution of tension within the narrative, emotional exploration of ambiguities within the diegesis, or identification with characters.

\begin{abstract}
"Gone Home requires you to use your own empathy to solve the puzzle of each family member's internal struggle" [13]

"Journey is a game purposefully designed to create argument and discussion. Most obviously people will argue over what exactly the games eponymous journey is all about. Who is the strange, robed figure you control and what is the significance of the shimmering mountain that seems to be his goal?" [20]
\end{abstract}

It is suggested here that these two types of challenge are antagonistic to each other. Where the player is called upon to navigate a complex task involving dexterity (such as in GOW3 or COD:MW2) the player is left with limited regard for or capacity to engage with anything other than the frustration/fiero cycle of hard fun. Conversely, the more avant-garde games examined here have simple controls and gameplay mechanics, making fewer demands in terms of functional challenge and therefore leaving the players mind freer to contemplate other areas of the game's possibility space [6]. Reviews of $S O: T L$ often pointed out how they found the combat repetitive and uninspiring but fully compensated for by the challenging narrative and character development.

"This could well be one of the most subversive shooters yet made. . your appetite for the emerging plot isn't stimulated by the standard stop-and-pop fare, which lacks a distinctive flavour of its own." [16]

It is worth considering that, had the combat been more intricate and challenging, the story and narrative may not have been experienced as fully. Papers Please is unusual in this regard in that it is the conflict between functional challenge and emotional challenge that provides the basis for the emotional experience. Whilst the mechanics are simple (as a passport control official you check papers at the border and accept or reject entrance), the choices presented in the game are not.

"There are moral decisions, like whether to separate a husband and wife whose paperwork doesn't match, or play white knight and turn a pimp away before he can get his hands on a girl who claims he tricked her over the border. The right answer may seem obvious, but every penalty that costs money means less food, heat, and medicine for the family back home - a family that has to stay alive..." [42]

"The only way to avoid failure is to remove emotion from the equation, and only look at the hard facts rather than the human realities. This job breeds hard men." [22]

\section{Value: Quality vs. Quantity}

Reviews of core games placed great importance on quantity of options and variations (or 'modes') of gameplay. Long passages were written on differing modes of gameplay (often with detailed descriptions), the number of weapons or abilities, maps, playable characters or the range of customisation options available. The length and/or variety of levels in the single player campaign is a significant factor in judging how valuable a purchase may be, as well as the depth and variety of any multi-player options available. 'Replayability' is a major concern. In summary - the number of hours of play that a game will provide is a key factor when deciding whether to make a purchase for a core game.

“...classic modes like Team Deathmatch and Gears favorites like Wingman on brilliant, varied maps... Overwhelming the human defenses as the Locust Horde is a wonderful Gears take on being the bad guys... This new Beast mode is as compelling to me as Horde mode... " [5]

This stands in contrast to the comments made about the more avant-garde games examined here. None of them offer much functional challenge and most are short in length with debatable or limited replay value (with the potential exception of Papers Please). However, this is frequently glossed over and forgotten in many reviews due to the emotional range, impact and depth shown by these games. What matters here is the intensity, novelty and quality of the emotional experience on offer, rather than length or replayability.

“....it's a triumphantly successful demonstration that narrative doesn't need to be funnelled down the barrel of a gun, balanced on the edge of a blade or relegated to a background cut scene for the sake of gameplay.” [33]

"However, in a mere hour and a half, Journey made more of an impact on my psyche than any game I have ever played." [14]

\section{Primary Emotion}

The emotions experienced by reviewers were strongly linked to the type of challenge that the game presented to the player.

Core games, with their reliance on hard fun to keep the player entertained elicited emotions often associated with action film and 'Hollywood blockbuster' tropes — with most reviewers having similar emotional experiences. This was mainly as a result of the intense action and functional challenge that these games require the player to engage in, as well as a dramatic narrative involved a large threat of some kind (e.g. the end of the world, the death of a loved one etc.) that the player must help avert. It is interesting to note that the quality of the 
narrative isn't expected to necessarily be very high in order to evoke these 'blockbuster drama' feelings.

"The story plays out like a Michael Bay film on steroids, filling the screen with explosions, military jargon, weapons of mass destruction...expect sweeping musical scores and pyrotechnics galore.” [12]

Emotions experienced by reviewers when playing the avantgarde games showed a greater range and were not the same for all players (cf. comparable emotional experiences between different reviews of core titles). Generally these involved a more reflective state of mind, contemplation and dealing with themes uncommon in core games.

Stronger and more emotive language was used to describe the depth and impact of the emotional experience of avant-garde games.

$$
\begin{aligned}
& \text { “... but more intriguing is its depiction of a family unit } \\
& \text { quietly tearing itself apart." [34] } \\
& \text { "Journey is an unforgettable experience. Even when the } \\
& \text { details fade, the emotions that it evoked will stay with us } \\
& \text { for years." [28] }
\end{aligned}
$$

\section{Ambiguity and Solitude}

Reviewers mention how curiosity is invoked by the lack of deliberate signposting and explicit communication of identity, plot or goals and objectives. These comments only occurred during reviews of avant-garde games.

"it's uncommonly enthralling. Its deliberate ambiguity brings on the urge to speculate on deeper meanings, but meaning here is bound to be personal, and best discovered for yourself." [15]

Some reviewers seem to suggest that feelings of loneliness and isolation in several of the avant-garde games examined here is conducive to emotional engagement.

"The perfect isolation of the island communicates a loneliness and sense of suspense that's far beyond what traditional games attempt." [27]

Tan has written about how an increased emotional investment by film viewers results in an increased emotional payback to the viewer [41] and Perron has explained how this may work for games players [31]. Other work has suggested that allowing a player to explore an emotional connection with an environment gives opportunity for a player's emotional intelligence to be stretched and strengthened [18], and that lack of definition for character motives and open-ended narratives are a unifying component of Art Cinema [7]. It is suggested here that ambiguity may well play a major role in the emotional impact of the avant-garde games studied in this paper. By leaving space for the player to think and contemplate - unburdened by the requirements of completing functional challenges, the player is better able to emotionally invest, and subsequently receive a greater emotional return, in the diegesis.

\section{Graphics and Sound}

Both visual and aural components are acknowledged by reviewers as being an important part of the game play experience, but different types of language are used to describe them across our two categories. Core games have their graphics described in terms of technical descriptions such as the detail of character and environmental models, lighting effects, realism and the quality of the textures. Sounds are described briefly, if mentioned at all, with statements usually referring to how the music underscores the intense action taking place and sounds grandiose and 'epic'.

"The environments feature some eye-popping visuals... The lighting engine has received a massive overhaul, the animations in the game are vastly improved, and the overall level of detail has jumped an order of magnitude.” [10]

This is in contrast to the avant-garde games examined here, where emotive language is frequently employed. Graphics are more likely to be described in terms of how they make the reviewer feel, rather than in terms of technological achievement. Sound equally is described in terms used to illustrate how the reviewer has been emotionally moved in ways other than raising levels of excitement.

“... set in one of those permanently autumnal corners of America where the late afternoon sun paints everything with a mixture of warmth and sorrow, and the game's artists wield this evocative palette like the old masters." [8]

“... thanks to a soundtrack by Jessica Curry that ebbs and flows brilliantly, overwhelming the senses with an atmosphere of unsettling, unseen dread." [17]

\section{DISCUSSION AND FUTURE WORK}

"As a medium defined by the word 'interactive' and shackled to the word 'entertainment', video games have long struggled with detaching themselves from violence and mechanical action in order to satisfy the latter of those terms." [33]

It is commonly assumed that for a game to be engaging it must provide some sort of challenge to the player and excel when they are about struggle.[21] However, conclusions drawn from this investigation suggests that challenge need not always take the form of logical puzzles to solve, obstacles to overcome or enemies to kill. The 'non-trivial traversal of the text' [1] can be done with purely cognitive effort instead of, or in addition to, completing challenges of dexterity and skill. The analysis carried out here illustrates that challenges can fall along a spectrum ranging from functional challenge at one end (requiring skill, dexterity or strategy to overcome environmental or AI obstacles using controls and mechanics) to emotional challenge at the other (requiring cognitive effort to deal with challenging material or comprehend ambiguous elements of the diegesis). This challenge spectrum has other attributes linked with the defining poles such as differing notions on what makes a game worthy of purchase, the role of graphics and sound in a game's appraisal and the role and type of emotion(s) experienced during gameplay. Ambiguity and 
solitude also appear to be commonly used devices to engineer a more emotive and reflective gameplay experience (as seen in VoEC, Gone Home, Dear Esther and in some cases Journey and Papers Please).

This paper was an attempt to elucidate whether successful avant-garde digital games have features in common to differentiate them from more traditional core games. The resultant findings, and particularly the notion of emotional and functional challenge, have important implications for the design and investigation of digital games in the future. An interesting extension of this work would be a comparison with results gained from using natural language processing using frameworks and methods already applied to videogames [32] [43].

If digital games are to continue to work towards engaging with a broader range of affect and a deeper resonance with the player's emotions similar to those enjoyed by readers of other art forms such as literature and film, then the functional and emotional challenge paradigm seems to be fertile ground for exploration by designers. Additionally, the tools of ambiguity and solitude warrant further investigation to see how different types and intensities of emotional challenge can be achieved, and conclusions made here can be used to work towards defining criteria for selecting other affective digital games for further analysis.

\section{ACKNOWLEDGEMENTS}

This work was supported by the EPSRC Centre for Doctoral Training in Intelligent Games and Game Intelligence.

\section{REFERENCES}

1. Aarseth, E. J. Cybertext: perspectives on ergodic literature. JHU Press, 1997.

2. Adams, A., Lunt, P., and Cairns, P. A Qualititative Approach to HCI Research. In Research Methods for Human-Computer Interaction, P. Cairns and A. Cox, Eds. Cambridge University Press, Cambridge, UK, 2008, 138-157.

3. Adams, E. W. Will computer games ever be a legitimate art form? Journal of Media Practice 7, 1 (2006), 67.

4. Alexander, L. 'Gamers' don't have to be your audience. 'Gamers' are over. http://www.gamasutra.com/view/ news $/ 224400 /$ Gamers_dont_have_to_be_your_ audience_Gamers_are_over.php., (Last Accessed 30/03/2015).

5. Biessener, A. Gears Of War 3 Review: A Blockbuster Finale - Gears of War 3 - Xbox 360 www.GameInformer.com.

http://www.gameinformer.com/games/gears_of_war_ 3/b/xbox360/archive/2011/09/15/ the-blockbuster-finale-millions-of-fans-are-craving 22 . aspx (Last Accessed 30/03/2015).

6. Bogost, I. Persuasive games: The expressive power of videogames. Mit Press, 2007.

7. Bordwell, D. The art cinema as a mode of film practice. Film Criticism 4, 1 (1979), 56-64.
8. Bramwell, T. The Vanishing of Ethan Carter Review. http: //www . eurogamer. net/articles/ 2014-09-30-the-vanishing-of-ethan-carter-review (Last Accessed 01/04/2015).

9. Buckland, J. Gone Home Review. http://www . atomicgamer.com/articles/1519/gone-home-review (Last Accessed 30/03/2015).

10. Burke, R. Gears of War 3 Review. http: //gamingtrend.com/game_reviews/gears-of-war-3/ (Last Accessed 30/03/2015).

11. Calvillo-Gámez, E. H., Cairns, P., and Cox, A. L. Assessing the core elements of the gaming experience. In Evaluating User Experience in Games. Springer, 2010, 47-71.

12. Deacon, A. Call of Duty: Modern Warfare 2 Review NZGamer.com. http://nzgamer.com/reviews/996/ call-of-duty-modern-warfare-2 .html (Last Accessed 30/03/2015).

13. Decker, L. Gone Home review - PC Gamer. http: //www.pcgamer.com/gone-home-review/ (Last Accessed 30/03/2015).

14. Deesing, J. Journey Review for PS3 - G4tv. http: //www.g4tv.com/games/ps3/64117/journey/review/ (Last Accessed 30/03/2015).

15. Douglas, J. Journey Review - GameSpot. http: //www.gamespot.com/reviews/journey-review/ 1900-6363693/ (Last Accessed 30/03/2015).

16. Edge. Spec Ops: The Line Review. http: //www . edge-online.com/review/spec-ops-line-review/ (Last Accessed 29/10/2014).

17. Grayson, N. Dear Esther Review. http: //pc.gamespy.com/pc/dear-esther/1218914p1.html (Last Accessed 01/04/2015).

18. Heron, M., and Belford, P. All of Your Co-workers are Gone: Story, Substance, and the Empathic Puzzler. Journal of Games Criticism 2, 1 (2015).

19. Ingenito, V. Destiny Review. http: //uk.ign.com/articles/2014/09/03/destiny-review (Last Accessed 01/04/2015).

20. Jenkins, D. Journey review destination unknown Metro News. http: //metro.co.uk/2012/03/02/ journey-review-destination-unknown-337875/ (Last Accessed 30/03/2015).

21. Jenkins, H. Games, the new lively art. Handbook of Computer Game Studies (2005), 175-192.

22. Jürgens, J. Papers Please Review. http: //www.thunderboltgames.com/review/papers-please (Last Accessed 01/04/2015).

23. Kushner, D. Masters of Doom: How two guys created an empire and transformed pop culture. Random House Incorporated, 2004. 
24. Lazarro, N. Four Keys to More Emotion Without Story. http://www.itu.dk/people/alec/articles/ xeodesign_whyweplaygames.pdf,(Last Accessed 30/03/2015).

25. Lee, T. Designing game narrative? http://hitboxteam.com/designing-game-narrative (Last Accessed 08/04/2014).

26. Lindley, C. A. The gameplay gestalt, narrative, and interactive storytelling. In CGDC Conf., Citeseer (2002).

27. MacDonald, K. Dear Esther Review. http://uk .ign. com/articles/2012/02/13/dear-esther-review (Last Accessed 01/04/2015).

28. McGarvey, S. Journey review | GamesRadar. http: //www.gamesradar.com/journey-review/ (Last Accessed 30/03/2015).

29. Neale, S. Art cinema as institution. Screen 22, 1 (1981), 11-39.

30. O'Brien, L. The future of video game storytelling. http://uk.ign.com/articles/2014/01/13/ the-future-of-video-game-storytelling (Last Accessed 08/04/2014).

31. Perron, B. A cognitive psychological approach to gameplay emotions. DiGRA 2005: Changing Views: Worlds in Play, 2005 International Conference (2005).

32. Raison, K., Tomuro, N., Lytinen, S., and Zagal, J. P. Extraction of user opinions by adjective-context co-clustering for game review texts. In Advances in Natural Language Processing. Springer, 2012, 289-299.

33. Sawrey, M. Gone Home - PC review at Thunderbolt. http:

//www.thunderboltgames.com/review/gone-home (Last Accessed 30/03/2015).

34. Schilling, C. Gone Home review - Telegraph. http: //www.telegraph.co.uk/technology/ video-games/video-game-reviews/10264724/ Gone-Home-review.html (Last Accessed 30/03/2015).

35. Schopenhauer, A. The world as will and representation, vol. 1. Courier Corporation, 2012.

36. Schrank, B. Avant-garde Videogames: Playing with Technoculture. MIT Press, 2014.

37. Sharp, J. Works of Game. MIT Press, 2015.

38. Smuts, A. Are Video Games Art? Contemporary Aesthetics 3 (2005).

39. Strauss, A., and Corbin, J. M. Basics of qualitative research: Grounded theory procedures and techniques. Sage Publications, Inc, 1990.

40. Stuart, K. Video games and art: why does the media get it so wrong? http://www.theguardian.com/ technology/gamesblog/2014/jan/08/ video-games-art-and-the-shock-of-the-new (Last Accessed 08/04/2014).
41. Tan, E. S. Emotion and the structure of narrative film: Film as an emotion machine. Routledge, 1995.

42. Whitehead, D. Papers Please Review. http: //www . eurogamer. net/articles/ 2013-08-09-papers-please-review (Last Accessed 01/04/2015).

43. Zagal, J. P., Tomuro, N., and Shepitsen, A. Natural language processing in game studies research: An overview. Simulation \& Gaming (2011), 1046878111422560.

\section{GAMES CITED}

Journey (2012), Sony Computer Entertainment Gone Home (2013), Fullbright

Gears of War 3 (2011), Microsoft Studios Call of Duty: Modern Warfare 2 (2009), Activision To The Moon (2011), Freebird Games Dear Esther (2012), The Chinese Room Vanishing of Ethan Carter (2014), The Astronauts Papers Please (2014), Lucas Pope Spec Ops: The Line (2012), 2K Games Bioshock (2007), 2K Games Last of Us (2013), Sony Computer Entertainment Grand Theft Auto V (2013), Rockstar Games Far Cry 4 (2014), Ubisoft Destiny (2014), Activision 